Nadira A Hatim

BDS, MSc (Prof.)

Mohammad A Mohi Al-Deen BDS, HD, (Prosthodontist)

\section{Patient's Satisfaction and Durability of Vertex Line Maxillary or Mandibular Complete Dentures}

\author{
Department of Prosthodontic \\ College of Dentistry, University of Mosul \\ Ninevah Health Directorate \\ Ministry of Health
}

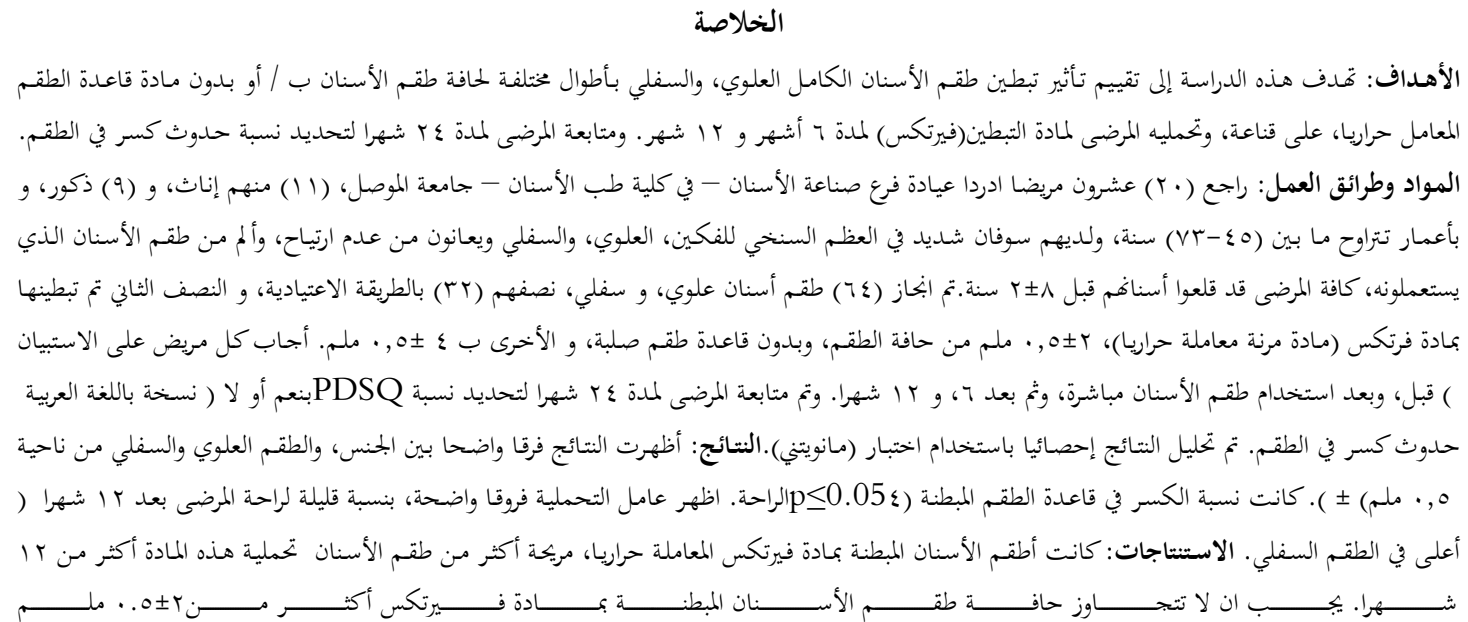

\title{
ABSTRACT
}

Aims: The aims of this study were to evaluate the effect of lining maxillary or mandibular complete denture with different length of flange periphery with or without heat cured denture base material on the Patient's Satisfaction and Durability of Vertex ${ }^{\circledR}$ Line materials. Materials and Methods: Twenty patients (11 Females, and 9 Males), age range 45-73 years with sever resorption completely edentulous arch (8 Patients) or arches (12 Patients) attend prosthodontic department clinic, College of Dentistry University of Mosul with complain of uncomfortable, soreness with previous prosthesis. All the

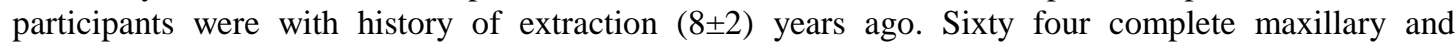
mandibular dentures were constructed, (32) complete denture was done in conventional method and the other half maxillary and mandibular dentures were lined with Vertex ${ }^{\circledR}$ (Heat cured liner), $2 \pm 0.5 \mathrm{~mm}$. of flange periphery without hard denture base material, and the other with $4 \pm 0.5 \mathrm{~mm}$. Each patient answers the Arabic version of (PDSQ) before and after insertion of the prosthesis and after 6months, and 12 months with follow up to 24months. Statistical analysis was done by using Mann-Whitney Test. Results: the results showed that a significant difference between gender, and significant differences between maxillary and mandibular denture for comfort. Durability factor showed significant differences with low percentage of patient comfort after 12 months $(p \leq 0.05)$. Percentage of cracks in hard denture base flange materials was higher in mandibular denture with $4 \mathrm{~mm}$. vertex lined materials. Conclusions: Complete denture lined with Vertex ${ }^{\circledR}$ heat cured material was more comfortable than conventional denture; Durability of this material was not more than 12 months. Vertex flange periphery should not more than $2 \pm 0.5 \mathrm{~mm}$.

Keywords: Vertex liner, Satisfaction, Durability of dentures.

Hatim NA, Mohi Al-Deen MA. Patient's Satisfaction and Durability of Vertex Line Maxillary or Mandibular Complete Dentures Al-Rafidain Dent J. 2014; 14(1):9-18.

Received: 2/4/2012

Sent to Referees: 8/4/2012

Accepted for Publication: 17/6/2012

\section{INTRODUCTION}

Soft denture liners are applied for denture wearers who cannot tolerate a hard-based denture due to a thin and nonresilient oral mucosa and/or severe alveolar resorption. Clinical success of the 
materials depends both on their viscoelastic properties and on durability. ${ }^{(1,}$ 2)

Denture lining materials have become important in dental prosthetic treatment. They are applied to the intaglio surface of dentures to achieve more equal force distribution, reduce localized pressure, and improve denture retention by engaging undercuts. ${ }^{(3)}$ Denture liners have been shown to increase a patient's level of comfort during mastication ${ }^{(4)}$. They provide comfort for patients who cannot tolerate occlusal pressures or who present alveolar ridge resorption, chronic soreness, and knife-edge ridges. ${ }^{(5)}$

Resilient denture liner may reduce trauma to mucosa compressed between the underlying bone spicules and a hard denture base. ${ }^{(6)}$

The aims of this study were to evaluate the effect of lining maxillary or mandibular complete denture with different length of flange periphery with or without heat cured denture base material for patients with severe bone resorption. And on the patient's satisfaction and durability of Vertex ${ }^{\circledR}$ Line material for periods, 6 months and 12 months.

\section{MATERIALS AND METHODS}

Sixty five patients were examined; only twenty patients (11 Females, and 9
Males) with age range between 45-73 years were selected from prosthodontic department clinic, College of Dentistry University of Mosul. Criteria for selection of patients are: The patients were complaining of sever bone resorption, height of ridge was about $6 \pm 0.5 \mathrm{~mm}$. was determined on stone cast from deepest point of vestibule to the crest of the ridge by using surveyor, presences of residual ridge soreness, and uncomfortable of previous hard acrylic resin complete denture base prosthesis. History of extraction was $8 \pm 2$ years ago, and systemically control.

Eight of twenty patients were completely edentulous arch (Maxillary or Mandibular arch), twelve of patients with completely edentulous arches. For each patient extra, intra oral examinations, and diagnostic cast were done.

Measurement of the residual ridge height of stone cast for each edentulous arch was done by using surveyor. The horizontal plane was recorded according to three points (Mid of the arch, and two posterior points just anterior to maxillary tuborosity and retromolar pad area of mandibular arches. The height of ridge $(6 \pm 0.5 \mathrm{~mm}$.) was measured at the midline anterior, and $1^{\text {st }}$ molar regions (Figure 1 ).
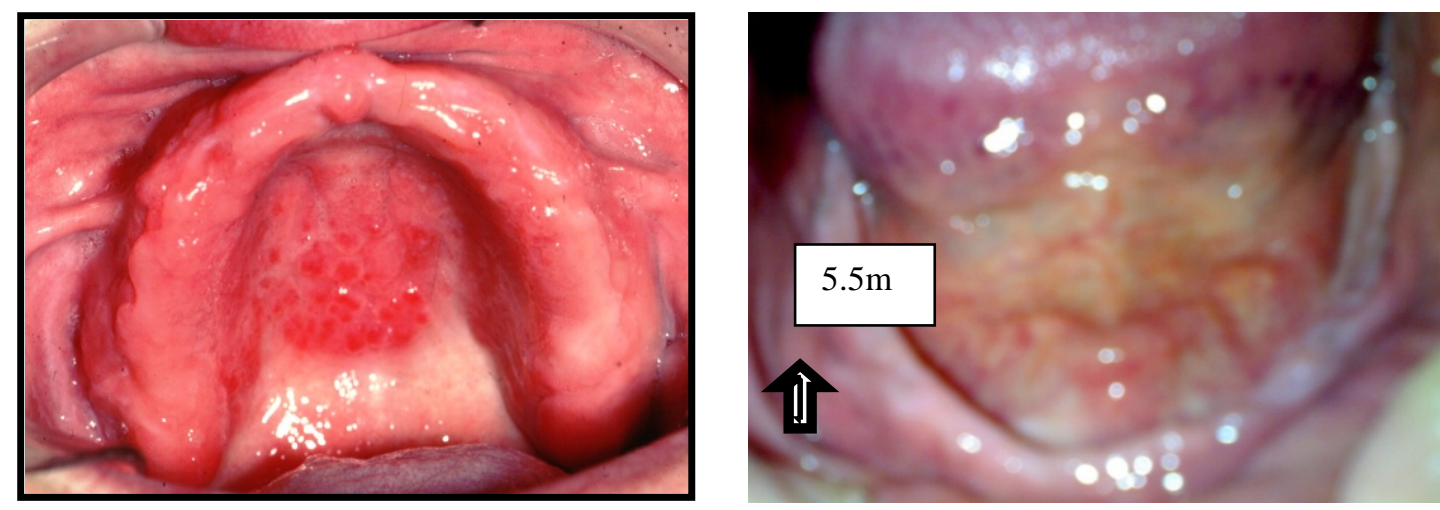

Figure (1): General soreness, and sever bone resorption

Thirty two conventional complete maxillary or mandibular dentures with balanced occlusion was constructed for all patients and used for one month as a control.Another (32) complete maxillary and/ or mandibular complete dentures (balanced occlusion ) were constructed for each patients lined with Vertex $^{\circledR}$ soft acrylic lining material (thickness of shellac base plate) according to manufacture instructions and divided into two groups. Lining space of sixteenth complete 
maxillary and/ or mandibular complete dentures (first group) was done by extending to $2 \pm 0.5 \mathrm{~mm}$. of the flange's length periphery without hard denture base material. While for the second group, lining was done by extending to $4 \pm 0.5 \mathrm{~mm}$. of the flange's length periphery without hard denture base material. ${ }^{(8)}$ And procedure of relining denture base done with monomer treatment for $180 \mathrm{sec}$ (Figures 2,3 and 4). ${ }^{(9)}$

(64) Maxillary or Mandibular Complete dentures lined with Vertex soft acrylic material

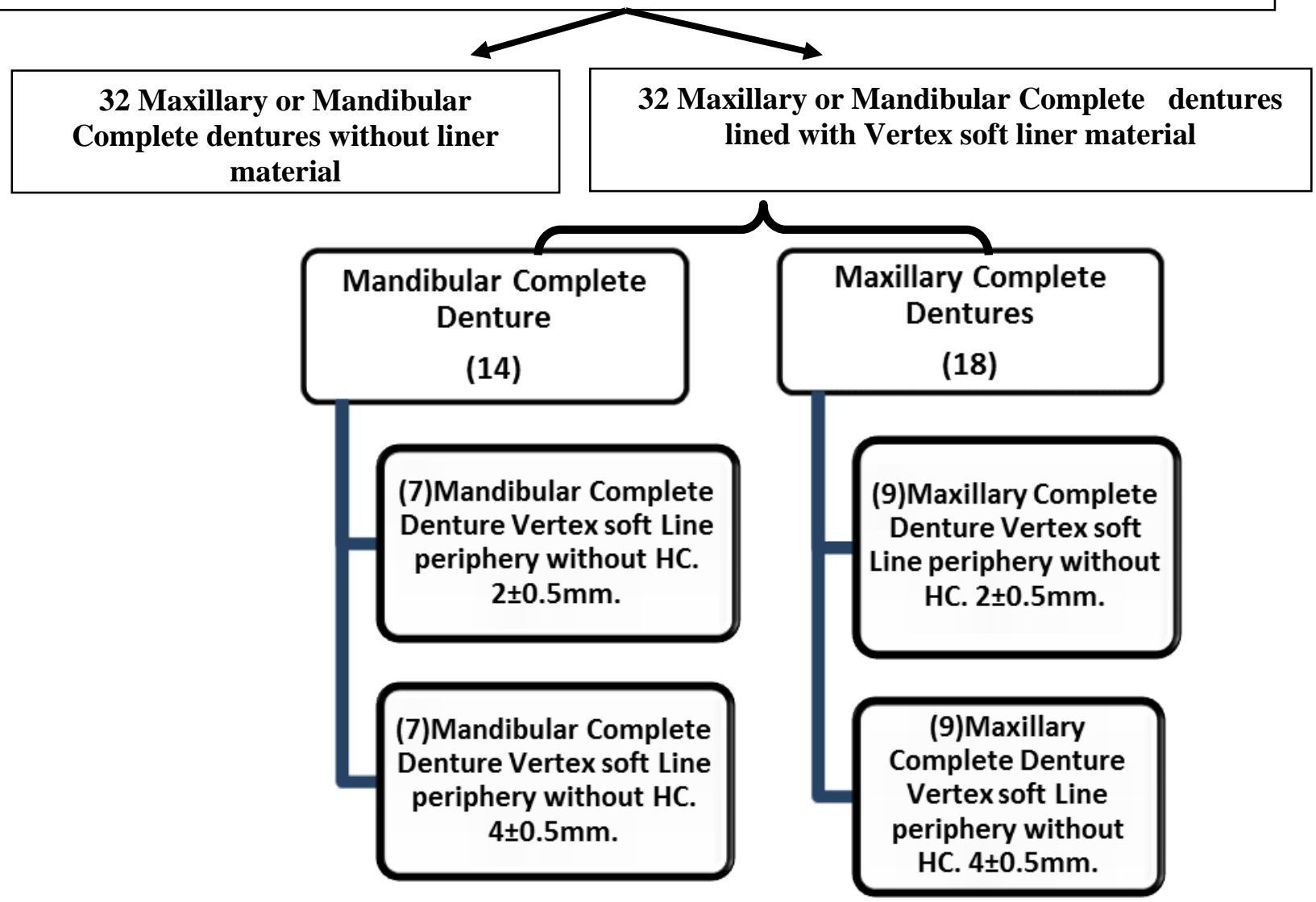

Figure (2): Experimental design of the study

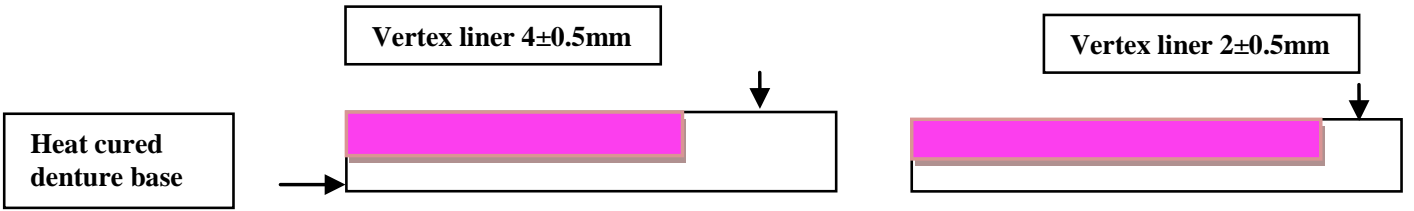

Figure (3): Schematic drawing mandibular complete denture lined with Vertex soft acrylic material at the periphery (2-4 mm. $\pm 0.5 \mathrm{~mm}$.) Without heat cured denture base material
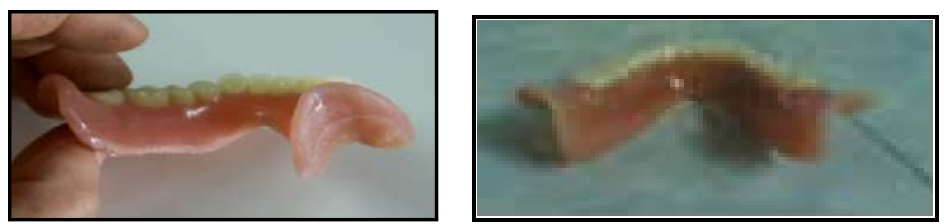

Figure (4): A: Vertex soft liner $2 \pm \mathbf{0 . 5} \mathrm{mm}$ periphery. B: Vertex soft liner $4 \pm \mathbf{0 . 5} \mathrm{mm}$ periphery.

At time of placement of complete maxillary or mandibular lined denture with vertex soft acrylic, a Denture patient satisfaction questionnaire (DPSQ) ${ }^{(10)}$
(Yes or No) was applied for each patient, and after 6months and 12 months in relation to the conventional previous complete denture worn by patient ${ }^{(10)}$ : 
1.

re you satisfied with your dentures?

2. Are you satisfied with the appearance of your dentures?

3. Are you satisfied with how well you upper or lower denture stays in place?

4. Are you satisfied with how well you chew food with your denture?

5. Are you satisfied with how well you speak with your dentures?

6. Are you satisfied with the comfort of your upper or lower denture?
7. How well other people (such as wife,A housebound, children, etc.) like your denture. Follow up of patients was continued to 24 months to asses fracture percentage of the dentures

\section{RESULTS}

Distribution of the sample according to gender and Mann-Whitney Test regarding each question showed that there was no significant difference $(\mathrm{p} \leq 0.05)$ except about question: How well other people (such as wife, housebound, children, etc.) like your denture (Table 1).

Table (1): Distribution of the sample according to gender and Mann-Whitney Test regarding each question

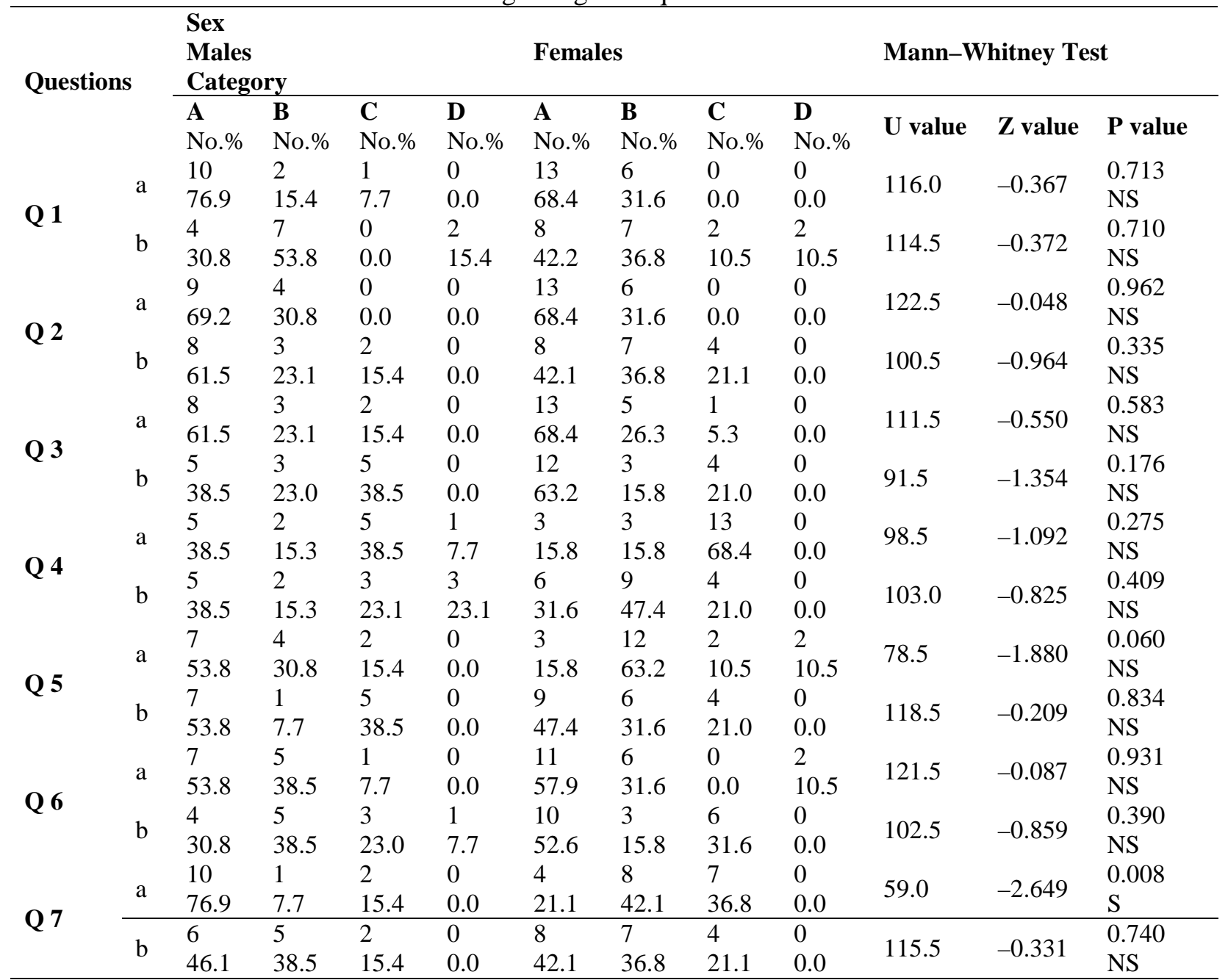

a: Before denture insertion; b: After denture insertion.NS: Not significant $(p>0.05)$; S: Significant $(p \leq 0.05)$.

Satisfaction with the comfort of upper or lower denture of the sample according to gender and Mann-Whitney showed that there was a significant difference $(\mathrm{p} \leq 0.05)$ for dentures with soft liner (6 and 12 months), (Table 2). 
Table (2): Distribution of the sample according to denture and Mann-Whitney Test regarding each question

\begin{tabular}{|c|c|c|c|c|c|c|c|c|c|c|c|c|}
\hline \multicolumn{2}{|c|}{ Questions } & \multicolumn{4}{|c|}{$\begin{array}{l}\text { Denture } \\
\text { Upper } \\
\text { Category }\end{array}$} & \multicolumn{4}{|c|}{ Lower } & \multicolumn{3}{|c|}{ Mann-Whitney Test } \\
\hline Quest & & $\begin{array}{l}\text { A } \\
\text { No. } \\
\%\end{array}$ & $\begin{array}{l}\text { B } \\
\text { No. } \\
\%\end{array}$ & $\begin{array}{l}\mathbf{C} \\
\text { No. } \\
\%\end{array}$ & $\begin{array}{l}\text { D } \\
\text { No. } \\
\%\end{array}$ & $\begin{array}{l}\text { A } \\
\text { No. } \\
\%\end{array}$ & $\begin{array}{l}\text { B } \\
\text { No. } \\
\%\end{array}$ & $\begin{array}{l}\mathbf{C} \\
\text { No. } \\
\%\end{array}$ & $\begin{array}{l}\text { D } \\
\text { No. } \\
\%\end{array}$ & $\begin{array}{l}\text { Ualue } \\
\text { valu }\end{array}$ & $\begin{array}{l}\mathrm{Z} \\
\text { value }\end{array}$ & $\underset{\text { value }}{\mathbf{p}}$ \\
\hline \multirow{2}{*}{ Q 1} & $\mathrm{a}$ & $\begin{array}{l}13 \\
72.2\end{array}$ & $\begin{array}{l}4 \\
22.2\end{array}$ & $\begin{array}{l}1 \\
5.6\end{array}$ & $\begin{array}{l}0 \\
0.0\end{array}$ & $\begin{array}{l}10 \\
71.4\end{array}$ & $\begin{array}{l}4 \\
28.6\end{array}$ & $\begin{array}{l}0 \\
0.0\end{array}$ & $\begin{array}{l}0 \\
0.0\end{array}$ & 125.0 & -0.048 & $\begin{array}{l}0.961 \\
\text { NS }\end{array}$ \\
\hline & $\mathrm{b}$ & $\begin{array}{l}8 \\
44.4\end{array}$ & $\begin{array}{l}7 \\
38.9\end{array}$ & $\begin{array}{l}1 \\
5.6\end{array}$ & $\begin{array}{l}2 \\
11.1\end{array}$ & $\begin{array}{l}4 \\
28.6\end{array}$ & $\begin{array}{l}7 \\
50.0\end{array}$ & $\begin{array}{l}1 \\
7.1\end{array}$ & $\begin{array}{l}2 \\
14.3\end{array}$ & 106.0 & -0.818 & $\begin{array}{l}0.413 \\
\text { NS }\end{array}$ \\
\hline \multirow{2}{*}{ Q 2} & $\mathrm{a}$ & $\begin{array}{l}13 \\
72.2\end{array}$ & $\begin{array}{l}5 \\
27.8\end{array}$ & $\begin{array}{l}0 \\
0.0\end{array}$ & $\begin{array}{l}0 \\
0.0\end{array}$ & & & $\begin{array}{l}0 \\
0.0\end{array}$ & $\begin{array}{l}0 \\
0.0\end{array}$ & 116.0 & -0.473 & $\begin{array}{l}0.636 \\
\text { NS }\end{array}$ \\
\hline & $\mathrm{b}$ & $\begin{array}{l}10 \\
55.5\end{array}$ & $\begin{array}{l}5 \\
27.8\end{array}$ & & $\begin{array}{l}0 \\
0.0\end{array}$ & $\begin{array}{l}6 \\
42.9\end{array}$ & & $\begin{array}{l}3 \\
21.4\end{array}$ & $\begin{array}{l}0 \\
0.0\end{array}$ & 110.0 & -0.664 & $\begin{array}{l}0.507 \\
\text { NS }\end{array}$ \\
\hline \multirow{2}{*}{ Q 3} & $\mathrm{a}$ & $\begin{array}{l}13 \\
72.2\end{array}$ & $\begin{array}{l}3 \\
16.7\end{array}$ & $\begin{array}{l}2 \\
11.1\end{array}$ & $\begin{array}{l}0 \\
0.0\end{array}$ & $\begin{array}{l}8 \\
57.2\end{array}$ & & $\begin{array}{l}1 \\
7.1\end{array}$ & $\begin{array}{l}0 \\
0.0\end{array}$ & 110.5 & -0.703 & $\begin{array}{l}0.482 \\
\text { NS }\end{array}$ \\
\hline & $\mathrm{b}$ & $\begin{array}{l}12 \\
66.6\end{array}$ & $\begin{array}{l}3 \\
16.7\end{array}$ & $\begin{array}{l}3 \\
16.7\end{array}$ & $\begin{array}{l}0 \\
0.0\end{array}$ & $\begin{array}{l}5 \\
35.7\end{array}$ & $\begin{array}{l}3 \\
21.4\end{array}$ & $\begin{array}{l}6 \\
42.9\end{array}$ & $\begin{array}{l}0 \\
0.0\end{array}$ & 82.5 & -1.823 & $\begin{array}{l}0.068 \\
\text { NS }\end{array}$ \\
\hline \multirow{2}{*}{ Q 4} & $\mathrm{a}$ & $\begin{array}{l}5 \\
27.8\end{array}$ & $\begin{array}{l}3 \\
16.7\end{array}$ & $\begin{array}{l}9 \\
50.0\end{array}$ & $\begin{array}{l}1 \\
5.5\end{array}$ & $\begin{array}{l}3 \\
21.4\end{array}$ & $\begin{array}{l}2 \\
14.3\end{array}$ & $\begin{array}{l}9 \\
64.3\end{array}$ & $\begin{array}{l}0 \\
0.0\end{array}$ & 119.0 & -0.297 & $\begin{array}{l}0.767 \\
\text { NS }\end{array}$ \\
\hline & $\mathrm{b}$ & $\begin{array}{l}7 \\
38.9\end{array}$ & $\begin{array}{l}6 \\
33.3\end{array}$ & $\begin{array}{l}4 \\
22.2\end{array}$ & $\begin{array}{l}1 \\
5.6\end{array}$ & $\begin{array}{l}4 \\
28.6\end{array}$ & $\begin{array}{l}5 \\
35.7\end{array}$ & $\begin{array}{l}3 \\
21.4\end{array}$ & $\begin{array}{l}2 \\
14.3\end{array}$ & 108.0 & -0.717 & $\begin{array}{l}0.473 \\
\text { NS }\end{array}$ \\
\hline \multirow{2}{*}{ Q 5} & $\mathrm{a}$ & $\begin{array}{l}6 \\
33.3\end{array}$ & $\begin{array}{l}9 \\
50.0\end{array}$ & $\begin{array}{l}2 \\
11.1\end{array}$ & $\begin{array}{l}1 \\
5.6\end{array}$ & $\begin{array}{l}4 \\
28.6\end{array}$ & $\begin{array}{l}7 \\
50.0\end{array}$ & $\begin{array}{l}2 \\
14.3\end{array}$ & $\begin{array}{l}1 \\
7.1\end{array}$ & 117.0 & -0.372 & $\begin{array}{l}0.710 \\
\text { NS }\end{array}$ \\
\hline & $\mathrm{b}$ & $\begin{array}{l}10 \\
55.6\end{array}$ & $\begin{array}{l}4 \\
22.2\end{array}$ & $\begin{array}{l}4 \\
22.2\end{array}$ & $\begin{array}{l}0 \\
0.0\end{array}$ & $\begin{array}{l}6 \\
42.9\end{array}$ & $\begin{array}{l}3 \\
21.4\end{array}$ & $\begin{array}{l}5 \\
35.7\end{array}$ & $\begin{array}{l}0 \\
0.0\end{array}$ & 106.0 & -0.827 & $\begin{array}{l}0.408 \\
\text { NS }\end{array}$ \\
\hline \multirow{2}{*}{ Q 6} & $\mathrm{a}$ & $\begin{array}{l}13 \\
72.2\end{array}$ & $\begin{array}{l}4 \\
22.2\end{array}$ & $\begin{array}{l}0 \\
0.0\end{array}$ & $\begin{array}{l}1 \\
5.6\end{array}$ & $\begin{array}{l}5 \\
35.8\end{array}$ & $\begin{array}{l}7 \\
50.0\end{array}$ & $\begin{array}{l}1 \\
7.1\end{array}$ & $\begin{array}{l}1 \\
7.1\end{array}$ & 80.0 & -1.976 & $\begin{array}{l}0.048 \\
S\end{array}$ \\
\hline & $\mathrm{b}$ & $\begin{array}{l}12 \\
66.6\end{array}$ & $\begin{array}{l}3 \\
16.7\end{array}$ & $\begin{array}{l}3 \\
16.7\end{array}$ & $\begin{array}{l}0 \\
0.0\end{array}$ & $\begin{array}{l}2 \\
14.3\end{array}$ & $\begin{array}{l}5 \\
35.7\end{array}$ & $\begin{array}{l}6 \\
42.9\end{array}$ & $\begin{array}{l}1 \\
7.1\end{array}$ & 55.5 & -2.856 & $\begin{array}{l}0.004 \\
\mathrm{~S}\end{array}$ \\
\hline \multirow{2}{*}{ Q 7} & $\mathrm{a}$ & $\begin{array}{l}9 \\
50.0\end{array}$ & $\begin{array}{l}4 \\
22.2\end{array}$ & $\begin{array}{l}5 \\
27.8\end{array}$ & $\begin{array}{l}0 \\
0.0\end{array}$ & $\begin{array}{l}5 \\
35.7\end{array}$ & $\begin{array}{l}5 \\
35.7\end{array}$ & $\begin{array}{l}4 \\
28.6\end{array}$ & $\begin{array}{l}0 \\
0.0\end{array}$ & 112.5 & -0.549 & $\begin{array}{l}0.583 \\
\text { NS }\end{array}$ \\
\hline & $\mathrm{b}$ & $\begin{array}{l}9 \\
50.0\end{array}$ & $\begin{array}{l}6 \\
33.3 \\
\end{array}$ & $\begin{array}{l}3 \\
16.7 \\
\end{array}$ & $\begin{array}{l}0 \\
0.0\end{array}$ & $\begin{array}{l}5 \\
35.7 \\
\end{array}$ & $\begin{array}{l}6 \\
42.9 \\
\end{array}$ & $\begin{array}{l}3 \\
21.4\end{array}$ & $\begin{array}{l}0 \\
0.0 \\
\end{array}$ & 108.0 & -0.738 & $\begin{array}{l}0.460 \\
\text { NS }\end{array}$ \\
\hline
\end{tabular}

a: Before denture insertion; b: After denture insertion with liner. NS: Not significant ( $p>0.05)$; S: Significant ( $p$

$$
\leq 0.05) \text {. }
$$

Mann-Whitney Test of Distribution of the sample according to duration wearing denture with soft liner (6 and 12 months) showed a significant differences for satisfaction with dentures, appearance, and How well other people (such as wife, housebound, children, etc.) like your denture? (Table 3) 
Table (3): Distribution of the sample according to duration of denture use and MannWhitney Test regarding each question

\begin{tabular}{|c|c|c|c|c|c|c|c|c|c|c|c|c|}
\hline \multirow{2}{*}{\multicolumn{2}{|c|}{ Questions }} & \multicolumn{4}{|c|}{$\begin{array}{l}\text { Duration of Denture Use } \\
6 \text { Months } \\
\text { Category }\end{array}$} & \multicolumn{4}{|c|}{12 Months } & \multicolumn{3}{|c|}{ Mann-Whitney Test } \\
\hline & & A & B & $\mathbf{C}$ & D & A & B & C & D & & & \\
\hline & & $\begin{array}{l}\text { No. } \\
\%\end{array}$ & $\begin{array}{l}\text { No. } \\
\%\end{array}$ & $\begin{array}{l}\text { No. } \\
\%\end{array}$ & $\begin{array}{l}\text { No. } \\
\%\end{array}$ & $\begin{array}{l}\text { No. } \\
\%\end{array}$ & $\begin{array}{l}\text { No. } \\
\%\end{array}$ & $\begin{array}{l}\text { No. } \\
\%\end{array}$ & $\begin{array}{l}\text { No. } \\
\%\end{array}$ & U value & $\begin{array}{l}\mathbf{L} \\
\text { value }\end{array}$ & $\begin{array}{l}\mathbf{p} \\
\text { value }\end{array}$ \\
\hline \multirow{3}{*}{ Q 1} & $\mathrm{a}$ & $\begin{array}{l}15 \\
68.2\end{array}$ & $\begin{array}{l}6 \\
27.3\end{array}$ & $\begin{array}{l}1 \\
4.5\end{array}$ & $\begin{array}{l}0 \\
0.0\end{array}$ & $\begin{array}{l}8 \\
80.0\end{array}$ & $\begin{array}{l}2 \\
20.0\end{array}$ & $\begin{array}{l}0 \\
0.0\end{array}$ & $\begin{array}{l}0 \\
0.0\end{array}$ & 96.0 & -0.727 & $\begin{array}{l}0.467 \\
\text { NS }\end{array}$ \\
\hline & & 12 & 6 & 2 & 2 & 8 & 2 & 0 & 0 & \multirow{2}{*}{58.0} & \multirow{2}{*}{-2.277} & 0.023 \\
\hline & b & 54.5 & 27.3 & 9.1 & 9.1 & 80.0 & 20.0 & 0.0 & 0.0 & & & S \\
\hline \multirow{2}{*}{ Q 2} & $\mathrm{a}$ & $\begin{array}{l}17 \\
77.3\end{array}$ & $\begin{array}{l}5 \\
22.7\end{array}$ & $\begin{array}{l}0 \\
0.0\end{array}$ & $\begin{array}{l}0 \\
0.0\end{array}$ & $\begin{array}{l}5 \\
50.0\end{array}$ & $\begin{array}{l}5 \\
50.0\end{array}$ & $\begin{array}{l}0 \\
0.0\end{array}$ & $\begin{array}{l}0 \\
0.0\end{array}$ & 80.0 & -1.518 & $\begin{array}{l}0.129 \\
\text { NS }\end{array}$ \\
\hline & $\mathrm{b}$ & $\begin{array}{l}14 \\
63.7\end{array}$ & $\begin{array}{l}7 \\
31.8\end{array}$ & $\begin{array}{l}1 \\
4.5\end{array}$ & $\begin{array}{l}0 \\
0.0\end{array}$ & $\begin{array}{l}2 \\
20.0\end{array}$ & $\begin{array}{l}3 \\
30.0\end{array}$ & $\begin{array}{l}5 \\
50.0\end{array}$ & $\begin{array}{l}0 \\
0.0\end{array}$ & 46.0 & -2.841 & $\begin{array}{l}0.004 \\
S\end{array}$ \\
\hline \multirow{2}{*}{ Q 3} & $\mathrm{a}$ & $\begin{array}{l}16 \\
72.7\end{array}$ & $\begin{array}{l}4 \\
18.2\end{array}$ & $\begin{array}{l}2 \\
9.1\end{array}$ & $\begin{array}{l}0 \\
0.0\end{array}$ & $\begin{array}{l}5 \\
50.0\end{array}$ & $\begin{array}{l}4 \\
40.0\end{array}$ & $\begin{array}{l}1 \\
10.0\end{array}$ & $\begin{array}{l}0 \\
0.0\end{array}$ & 87.0 & -1.116 & $\begin{array}{l}0.264 \\
\text { NS }\end{array}$ \\
\hline & $\mathrm{b}$ & $\begin{array}{l}12 \\
54.5\end{array}$ & $\begin{array}{l}4 \\
18.2\end{array}$ & $\begin{array}{l}6 \\
27.3\end{array}$ & $\begin{array}{l}0 \\
0.0\end{array}$ & $\begin{array}{l}5 \\
50.0\end{array}$ & $\begin{array}{l}2 \\
20.0\end{array}$ & $\begin{array}{l}3 \\
30.0\end{array}$ & $\begin{array}{l}0 \\
0.0\end{array}$ & 105.0 & -0.224 & $\begin{array}{l}0.823 \\
\text { NS }\end{array}$ \\
\hline \multirow{2}{*}{ Q 4} & $\mathrm{a}$ & $\begin{array}{l}5 \\
22.8\end{array}$ & $\begin{array}{l}3 \\
13.6\end{array}$ & $\begin{array}{l}13 \\
59.1\end{array}$ & $\begin{array}{l}1 \\
4.5\end{array}$ & $\begin{array}{l}3 \\
30.0\end{array}$ & $\begin{array}{l}2 \\
20.0\end{array}$ & $\begin{array}{l}5 \\
50.0\end{array}$ & $\begin{array}{l}0 \\
0.0\end{array}$ & 93.0 & -0.771 & $\begin{array}{l}0.441 \\
\text { NS }\end{array}$ \\
\hline & $\mathrm{b}$ & $\begin{array}{l}8 \\
36.4\end{array}$ & $\begin{array}{l}8 \\
36.4\end{array}$ & $\begin{array}{l}3 \\
13.6\end{array}$ & $\begin{array}{l}3 \\
13.6\end{array}$ & $\begin{array}{l}3 \\
30.0\end{array}$ & $\begin{array}{l}3 \\
30.0\end{array}$ & $\begin{array}{l}4 \\
40.0\end{array}$ & $\begin{array}{l}0 \\
0.0\end{array}$ & 102.0 & -0.341 & $\begin{array}{l}0.733 \\
\text { NS }\end{array}$ \\
\hline \multirow{2}{*}{ Q 5} & $\mathrm{a}$ & $\begin{array}{l}7 \\
31.8\end{array}$ & $\begin{array}{l}11 \\
50.0\end{array}$ & $\begin{array}{l}2 \\
9.1\end{array}$ & $\begin{array}{l}2 \\
9.1\end{array}$ & $\begin{array}{l}3 \\
30.0\end{array}$ & $\begin{array}{l}5 \\
50.0\end{array}$ & $\begin{array}{l}2 \\
20.0\end{array}$ & $\begin{array}{l}0 \\
0.0\end{array}$ & 109.0 & -0.044 & $\begin{array}{l}0.965 \\
\text { NS }\end{array}$ \\
\hline & $\mathrm{b}$ & $\begin{array}{l}12 \\
54.6\end{array}$ & $\begin{array}{l}5 \\
22.7\end{array}$ & $\begin{array}{l}5 \\
22.7\end{array}$ & $\begin{array}{l}0 \\
0.0\end{array}$ & $\begin{array}{l}4 \\
40.0\end{array}$ & $\begin{array}{l}2 \\
20.0\end{array}$ & $\begin{array}{l}4 \\
40.0\end{array}$ & $\begin{array}{l}0 \\
0.0\end{array}$ & 89.0 & -0.930 & $\begin{array}{l}0.352 \\
\text { NS }\end{array}$ \\
\hline \multirow{2}{*}{ Q 6} & $\mathrm{a}$ & $\begin{array}{l}12 \\
54.5\end{array}$ & $\begin{array}{l}7 \\
31.9\end{array}$ & $\begin{array}{l}1 \\
4.5\end{array}$ & $\begin{array}{l}2 \\
9.1\end{array}$ & $\begin{array}{l}6 \\
60.0\end{array}$ & $\begin{array}{l}4 \\
40.0\end{array}$ & $\begin{array}{l}0 \\
0.0\end{array}$ & $\begin{array}{l}0 \\
0.0\end{array}$ & 98.0 & -0.552 & $\begin{array}{l}0.581 \\
\text { NS }\end{array}$ \\
\hline & $\mathrm{b}$ & $\begin{array}{l}12 \\
54.5\end{array}$ & $\begin{array}{l}5 \\
22.7\end{array}$ & $\begin{array}{l}4 \\
18.3\end{array}$ & $\begin{array}{l}1 \\
4.5\end{array}$ & $\begin{array}{l}2 \\
20.0\end{array}$ & $\begin{array}{l}3 \\
30.0\end{array}$ & $\begin{array}{l}5 \\
50.0\end{array}$ & $\begin{array}{l}0 \\
0.0\end{array}$ & 69.5 & -1.756 & $\begin{array}{l}0.079 \\
\text { NS }\end{array}$ \\
\hline \multirow{2}{*}{ Q 7} & $\mathrm{a}$ & $\begin{array}{l}11 \\
50.0\end{array}$ & $\begin{array}{l}3 \\
13.6\end{array}$ & $\begin{array}{l}8 \\
36.4\end{array}$ & $\begin{array}{l}0 \\
0.0\end{array}$ & $\begin{array}{l}3 \\
30.0\end{array}$ & $\begin{array}{l}6 \\
60.0\end{array}$ & $\begin{array}{l}1 \\
10.0\end{array}$ & $\begin{array}{l}0 \\
0.0\end{array}$ & 109.5 & -0.022 & $\begin{array}{l}0.983 \\
\text { NS }\end{array}$ \\
\hline & $\mathrm{b}$ & $\begin{array}{l}13 \\
59.1 \\
\end{array}$ & $\begin{array}{l}7 \\
31.8 \\
\end{array}$ & $\begin{array}{l}2 \\
9.1 \\
\end{array}$ & $\begin{array}{l}0 \\
0.0 \\
\end{array}$ & $\begin{array}{l}1 \\
10.0 \\
\end{array}$ & $\begin{array}{l}5 \\
50.0 \\
\end{array}$ & $\begin{array}{l}4 \\
40.0 \\
\end{array}$ & $\begin{array}{l}0 \\
0.0 \\
\end{array}$ & 47.0 & -2.766 & $\begin{array}{l}0.006 \\
\mathrm{~S} \\
\end{array}$ \\
\hline
\end{tabular}

a: Before denture insertion; b: After denture insertion. NS: Not significant $(p>0.05)$; S: Significant $(p$ $\leq 0.05)$.

Distribution of the sample according to flange's length of periphery without hard denture base $2 \pm 0.5 \mathrm{~mm}$. and $4 \pm 0.5 \mathrm{~mm}$., and Mann-Whitney Test regarding each question (Upper and lower dentures for 6 and 12 months) showed no significant differences ( Tables 4, and 5). 
Table (4): Distribution of the sample according to Periphery height and Mann-Whitney Test regarding each question (Upper denture)

\begin{tabular}{|c|c|c|c|c|c|c|c|c|c|c|c|c|}
\hline \multicolumn{2}{|c|}{ Questions } & \multicolumn{4}{|c|}{$\begin{array}{l}\text { Periphery height } \\
2 \text { mm } \\
\text { Category }\end{array}$} & \multicolumn{4}{|c|}{$4 \mathrm{~mm}$} & \multicolumn{3}{|c|}{ Mann-Whitney Test } \\
\hline Ques & & $\begin{array}{l}\text { A } \\
\text { No. } \\
\%\end{array}$ & $\begin{array}{l}\text { B } \\
\text { No. } \\
\%\end{array}$ & $\begin{array}{l}\mathbf{C} \\
\text { No. } \\
\%\end{array}$ & $\begin{array}{l}\text { D } \\
\text { No. } \\
\%\end{array}$ & $\begin{array}{l}\text { A } \\
\text { No. } \\
\%\end{array}$ & $\begin{array}{l}\text { B } \\
\text { No. } \\
\%\end{array}$ & $\begin{array}{l}\mathbf{C} \\
\text { No. } \\
\%\end{array}$ & $\begin{array}{l}\text { D } \\
\text { No. } \\
\%\end{array}$ & U value & $\begin{array}{l}\mathrm{Z} \\
\text { value }\end{array}$ & $\begin{array}{l}p \\
\text { value }\end{array}$ \\
\hline \multirow{2}{*}{ Q 1} & $\mathrm{a}$ & $\begin{array}{l}7 \\
77.8\end{array}$ & $\begin{array}{l}2 \\
22.2\end{array}$ & $\begin{array}{l}0 \\
0.0\end{array}$ & $\begin{array}{l}0 \\
0.0\end{array}$ & $\begin{array}{l}6 \\
66.7\end{array}$ & $\begin{array}{l}2 \\
22.2\end{array}$ & $\begin{array}{l}1 \\
11.1\end{array}$ & $\begin{array}{l}0 \\
0.0\end{array}$ & 35.00 & -0.620 & $\begin{array}{l}0.535 \\
\text { NS }\end{array}$ \\
\hline & $\mathrm{b}$ & $\begin{array}{l}5 \\
55.6\end{array}$ & $\begin{array}{l}2 \\
22.2\end{array}$ & $\begin{array}{l}1 \\
11.1\end{array}$ & $\begin{array}{l}1 \\
11.1\end{array}$ & $\begin{array}{l}3 \\
33.3\end{array}$ & $\begin{array}{l}5 \\
55.6\end{array}$ & $\begin{array}{l}0 \\
0.0\end{array}$ & $\begin{array}{l}1 \\
11.1\end{array}$ & 35.00 & -0.525 & $\begin{array}{l}0.599 \\
\text { NS }\end{array}$ \\
\hline \multirow[t]{2}{*}{ Q 2} & $\mathrm{a}$ & $\begin{array}{l}7 \\
77.8\end{array}$ & $\begin{array}{l}2 \\
22.2\end{array}$ & $\begin{array}{l}0 \\
0.0\end{array}$ & $\begin{array}{l}0 \\
0.0\end{array}$ & $\begin{array}{l}6 \\
66.7\end{array}$ & $\begin{array}{l}3 \\
33.3\end{array}$ & $\begin{array}{l}0 \\
0.0\end{array}$ & $\begin{array}{l}0 \\
0.0\end{array}$ & 36.00 & -0.511 & $\begin{array}{l}0.609 \\
\text { NS }\end{array}$ \\
\hline & $\mathrm{b}$ & $\begin{array}{l}6 \\
66.7\end{array}$ & $\begin{array}{l}3 \\
33.3\end{array}$ & $\begin{array}{l}0 \\
0.0\end{array}$ & $\begin{array}{l}0 \\
0.0\end{array}$ & $\begin{array}{l}4 \\
44.5\end{array}$ & $\begin{array}{l}2 \\
22.2\end{array}$ & $\begin{array}{l}3 \\
33.3\end{array}$ & $\begin{array}{l}0 \\
0.0\end{array}$ & 27.00 & -1.329 & $\begin{array}{l}0.184 \\
\text { NS }\end{array}$ \\
\hline \multirow{2}{*}{ Q 3} & a & $\begin{array}{l}7 \\
77.8\end{array}$ & $\begin{array}{l}1 \\
11.1\end{array}$ & $\begin{array}{l}1 \\
11.1\end{array}$ & $\begin{array}{l}0 \\
0.0\end{array}$ & $\begin{array}{l}6 \\
66.7\end{array}$ & $\begin{array}{l}2 \\
22.2\end{array}$ & $\begin{array}{l}1 \\
11.1\end{array}$ & $\begin{array}{l}0 \\
0.0\end{array}$ & 36.50 & -0.449 & $\begin{array}{l}0.654 \\
\text { NS }\end{array}$ \\
\hline & $\mathrm{b}$ & $\begin{array}{l}7 \\
77.8\end{array}$ & $\begin{array}{l}0 \\
0.0\end{array}$ & $\begin{array}{l}2 \\
22.2\end{array}$ & $\begin{array}{l}0 \\
0.0\end{array}$ & $\begin{array}{l}5 \\
55.6\end{array}$ & $\begin{array}{l}3 \\
33.3\end{array}$ & $\begin{array}{l}1 \\
11.1\end{array}$ & $\begin{array}{l}0 \\
0.0\end{array}$ & 34.50 & -0.635 & $\begin{array}{l}0.526 \\
\text { NS }\end{array}$ \\
\hline \multirow{2}{*}{ Q 4} & a & $\begin{array}{l}4 \\
44.4\end{array}$ & $\begin{array}{l}0 \\
0.0\end{array}$ & $\begin{array}{l}5 \\
55.6\end{array}$ & $\begin{array}{l}0 \\
0.0\end{array}$ & $\begin{array}{l}1 \\
11.1\end{array}$ & $\begin{array}{l}3 \\
33.3\end{array}$ & $\begin{array}{l}4 \\
44.5\end{array}$ & $\begin{array}{l}1 \\
11.1\end{array}$ & 32.00 & -0.813 & $\begin{array}{l}0.416 \\
\text { NS }\end{array}$ \\
\hline & $\mathrm{b}$ & $\begin{array}{l}4 \\
44.5\end{array}$ & $\begin{array}{l}3 \\
33.3\end{array}$ & $\begin{array}{l}1 \\
11.1\end{array}$ & $\begin{array}{l}1 \\
11.1\end{array}$ & $\begin{array}{l}3 \\
33.3\end{array}$ & $\begin{array}{l}3 \\
33.3\end{array}$ & $\begin{array}{l}3 \\
33.3\end{array}$ & $\begin{array}{l}0 \\
0.0\end{array}$ & 36.00 & -0.420 & $\begin{array}{l}0.675 \\
\text { NS }\end{array}$ \\
\hline \multirow{2}{*}{ Q 5} & a & $\begin{array}{l}4 \\
44.4\end{array}$ & $\begin{array}{l}4 \\
44.4\end{array}$ & $\begin{array}{l}1 \\
11.2\end{array}$ & $\begin{array}{l}0 \\
0.0\end{array}$ & $\begin{array}{l}2 \\
22.2\end{array}$ & $\begin{array}{l}5 \\
55.6\end{array}$ & $\begin{array}{l}1 \\
11.1\end{array}$ & $\begin{array}{l}1 \\
11.1\end{array}$ & 29.50 & -1.060 & $\begin{array}{l}0.289 \\
\text { NS }\end{array}$ \\
\hline & $\mathrm{b}$ & $\begin{array}{l}5 \\
55.6\end{array}$ & $\begin{array}{l}2 \\
22.2\end{array}$ & $\begin{array}{l}2 \\
22.2\end{array}$ & $\begin{array}{l}0 \\
0.0\end{array}$ & $\begin{array}{l}5 \\
55.6\end{array}$ & $\begin{array}{l}2 \\
22.2\end{array}$ & $\begin{array}{l}2 \\
22.2\end{array}$ & $\begin{array}{l}0 \\
0.0\end{array}$ & 40.50 & 0.000 & $\begin{array}{l}1.000 \\
\text { NS }\end{array}$ \\
\hline \multirow{2}{*}{ Q 6} & a & $\begin{array}{l}8 \\
88.9\end{array}$ & $\begin{array}{l}1 \\
11.1\end{array}$ & $\begin{array}{l}0 \\
0.0\end{array}$ & $\begin{array}{l}0 \\
0.0\end{array}$ & $\begin{array}{l}5 \\
55.6\end{array}$ & $\begin{array}{l}3 \\
33.3\end{array}$ & $\begin{array}{l}0 \\
0.0\end{array}$ & $\begin{array}{l}1 \\
11.1\end{array}$ & 26.50 & -1.578 & $\begin{array}{l}0.115 \\
\text { NS }\end{array}$ \\
\hline & $\mathrm{b}$ & $\begin{array}{l}7 \\
77.8\end{array}$ & $\begin{array}{l}1 \\
11.1\end{array}$ & $\begin{array}{l}1 \\
11.1\end{array}$ & $\begin{array}{l}0 \\
0.0\end{array}$ & $\begin{array}{l}5 \\
55.6\end{array}$ & $\begin{array}{l}2 \\
22.2\end{array}$ & $\begin{array}{l}2 \\
22.2\end{array}$ & $\begin{array}{l}0 \\
0.0\end{array}$ & 31.50 & -0.952 & $\begin{array}{l}0.341 \\
\text { NS }\end{array}$ \\
\hline \multirow{2}{*}{ Q 7} & $\mathrm{a}$ & $\begin{array}{l}5 \\
55.6 \\
\end{array}$ & $\begin{array}{l}1 \\
11.1\end{array}$ & $\begin{array}{l}3 \\
33.3 \\
\end{array}$ & $\begin{array}{l}0 \\
0.0\end{array}$ & $\begin{array}{l}4 \\
44.5 \\
\end{array}$ & $\begin{array}{l}3 \\
33.3 \\
\end{array}$ & $\begin{array}{l}2 \\
22.2\end{array}$ & $\begin{array}{l}0 \\
0.0\end{array}$ & 39.50 & -0.096 & $\begin{array}{l}0.923 \\
\text { NS }\end{array}$ \\
\hline & $\mathrm{b}$ & $\begin{array}{l}5 \\
55.6 \\
\end{array}$ & $\begin{array}{l}3 \\
33.3 \\
\end{array}$ & $\begin{array}{l}1 \\
11.1 \\
\end{array}$ & $\begin{array}{l}0 \\
0.0 \\
\end{array}$ & $\begin{array}{l}4 \\
44.5 \\
\end{array}$ & $\begin{array}{l}3 \\
33.3 \\
\end{array}$ & $\begin{array}{l}2 \\
22.2 \\
\end{array}$ & $\begin{array}{l}0 \\
0.0 \\
\end{array}$ & 34.50 & -0.579 & $\begin{array}{l}0.562 \\
\text { NS }\end{array}$ \\
\hline
\end{tabular}

a: Before denture insertion; b: After denture insertion. NS: Not significant $(p>0.05)$. 
Table (5): Distribution of the sample according to Periphery height and Mann-Whitney Test regarding each question (Lower denture)

\begin{tabular}{|c|c|c|c|c|c|c|c|c|c|c|c|c|}
\hline \multirow{2}{*}{\multicolumn{2}{|c|}{ Questions }} & \multicolumn{4}{|c|}{$\begin{array}{l}\text { Periphery height } \\
2 \text { mm } \\
\text { Category }\end{array}$} & \multicolumn{4}{|c|}{$4 \mathrm{~mm}$} & \multicolumn{3}{|c|}{ Mann-Whitney Test } \\
\hline & & A & B & C & D & $\mathbf{A}$ & B & C & D & & & \\
\hline & & $\begin{array}{l}\text { No. } \\
\%\end{array}$ & $\begin{array}{l}\text { No. } \\
\%\end{array}$ & $\begin{array}{l}\text { No. } \\
\%\end{array}$ & $\begin{array}{l}\text { No. } \\
\%\end{array}$ & $\begin{array}{l}\text { No. } \\
\%\end{array}$ & $\begin{array}{l}\text { No. } \\
\%\end{array}$ & $\begin{array}{l}\text { No. } \\
\%\end{array}$ & $\begin{array}{l}\text { No. } \\
\%\end{array}$ & $\mathrm{U}$ value & $\begin{array}{l}\mathbf{Z} \\
\text { value }\end{array}$ & $\begin{array}{l}\text { P } \\
\text { value }\end{array}$ \\
\hline \multirow{3}{*}{ Q 1} & $\mathrm{a}$ & $\begin{array}{l}5 \\
71.4\end{array}$ & $\begin{array}{l}2 \\
28.6\end{array}$ & $\begin{array}{l}0 \\
0.0\end{array}$ & $\begin{array}{l}0 \\
0.0\end{array}$ & $\begin{array}{l}5 \\
71.4\end{array}$ & $\begin{array}{l}2 \\
28.6\end{array}$ & $\begin{array}{l}0 \\
0.0\end{array}$ & $\begin{array}{l}0 \\
0.0\end{array}$ & 24.50 & 0.000 & $\begin{array}{l}1.000 \\
\text { NS }\end{array}$ \\
\hline & $b$ & 2 & 4 & 1 & 0 & 2 & 3 & 1 & 1 & & & 0.782 \\
\hline & D & 28.6 & 57.1 & 14.3 & 0.0 & 28.6 & 42.8 & 14.3 & 14.3 & 22.50 & -0.277 & NS \\
\hline \multirow{2}{*}{ Q 2} & $\mathrm{a}$ & $\begin{array}{l}5 \\
71.4\end{array}$ & $\begin{array}{l}2 \\
28.6\end{array}$ & $\begin{array}{l}0 \\
0.0\end{array}$ & $\begin{array}{l}0 \\
0.0\end{array}$ & $\begin{array}{l}4 \\
57.1\end{array}$ & $\begin{array}{l}3 \\
42.9\end{array}$ & $\begin{array}{l}0 \\
0.0\end{array}$ & $\begin{array}{l}0 \\
0.0\end{array}$ & 21.00 & -0.537 & $\begin{array}{l}0.591 \\
\text { NS }\end{array}$ \\
\hline & $\mathrm{b}$ & $\begin{array}{l}3 \\
42.8\end{array}$ & $\begin{array}{l}2 \\
28.6\end{array}$ & $\begin{array}{l}2 \\
28.6\end{array}$ & $\begin{array}{l}0 \\
0.0\end{array}$ & $\begin{array}{l}3 \\
42.9\end{array}$ & $\begin{array}{l}3 \\
42.9\end{array}$ & $\begin{array}{l}1 \\
14.2\end{array}$ & $\begin{array}{l}0 \\
0.0\end{array}$ & 22.50 & -0.274 & $\begin{array}{l}0.784 \\
\text { NS }\end{array}$ \\
\hline \multirow{2}{*}{ Q 3} & $\mathrm{a}$ & $\begin{array}{l}3 \\
42.9\end{array}$ & $\begin{array}{l}4 \\
57.1\end{array}$ & $\begin{array}{l}0 \\
0.0\end{array}$ & $\begin{array}{l}0 \\
0.0\end{array}$ & $\begin{array}{l}5 \\
71.4\end{array}$ & $\begin{array}{l}1 \\
14.3\end{array}$ & $\begin{array}{l}1 \\
14.3\end{array}$ & $\begin{array}{l}0 \\
0.0\end{array}$ & 19.50 & -0.727 & $\begin{array}{l}0.467 \\
\text { NS }\end{array}$ \\
\hline & $\mathrm{b}$ & $\begin{array}{l}4 \\
57.1\end{array}$ & $\begin{array}{l}3 \\
42.9\end{array}$ & $\begin{array}{l}0 \\
0.0\end{array}$ & $\begin{array}{l}0 \\
0.0\end{array}$ & $\begin{array}{l}1 \\
14.2\end{array}$ & $\begin{array}{l}3 \\
42.9\end{array}$ & $\begin{array}{l}3 \\
42.9\end{array}$ & $\begin{array}{l}0 \\
0.0\end{array}$ & 18.50 & -0.822 & $\begin{array}{l}0.411 \\
\text { NS }\end{array}$ \\
\hline \multirow{2}{*}{ Q 4} & $\mathrm{a}$ & $\begin{array}{l}1 \\
14.3\end{array}$ & $\begin{array}{l}2 \\
28.6\end{array}$ & $\begin{array}{l}4 \\
57.1\end{array}$ & $\begin{array}{l}0 \\
0.0\end{array}$ & $\begin{array}{l}2 \\
28.6\end{array}$ & $\begin{array}{l}0 \\
0.0\end{array}$ & $\begin{array}{l}5 \\
71.4\end{array}$ & $\begin{array}{l}0 \\
0.0\end{array}$ & 23.00 & -0.225 & $\begin{array}{l}0.822 \\
\text { NS }\end{array}$ \\
\hline & $\mathrm{b}$ & $\begin{array}{l}2 \\
28.6\end{array}$ & $\begin{array}{l}2 \\
28.6\end{array}$ & $\begin{array}{l}2 \\
28.6\end{array}$ & $\begin{array}{l}1 \\
14.2\end{array}$ & $\begin{array}{l}2 \\
28.6\end{array}$ & $\begin{array}{l}3 \\
42.8\end{array}$ & $\begin{array}{l}1 \\
14.3\end{array}$ & $\begin{array}{l}1 \\
14.3\end{array}$ & 22.50 & -0.266 & $\begin{array}{l}0.790 \\
\text { NS }\end{array}$ \\
\hline \multirow{2}{*}{ Q 5} & $\mathrm{a}$ & $\begin{array}{l}2 \\
28.6\end{array}$ & $\begin{array}{l}3 \\
42.8\end{array}$ & $\begin{array}{l}1 \\
14.3\end{array}$ & $\begin{array}{l}1 \\
14.3\end{array}$ & $\begin{array}{l}2 \\
28.6\end{array}$ & $\begin{array}{l}4 \\
57.1\end{array}$ & $\begin{array}{l}1 \\
14.3\end{array}$ & $\begin{array}{l}0 \\
0.0\end{array}$ & 21.50 & -0.415 & $\begin{array}{l}0.678 \\
\text { NS }\end{array}$ \\
\hline & $\mathrm{b}$ & $\begin{array}{l}3 \\
42.9\end{array}$ & $\begin{array}{l}1 \\
14.2\end{array}$ & $\begin{array}{l}3 \\
42.9\end{array}$ & $\begin{array}{l}0 \\
0.0\end{array}$ & $\begin{array}{l}3 \\
42.8\end{array}$ & $\begin{array}{l}2 \\
28.6\end{array}$ & $\begin{array}{l}2 \\
28.6\end{array}$ & $\begin{array}{l}0 \\
0.0\end{array}$ & 22.50 & -0.274 & $\begin{array}{l}0.784 \\
\text { NS }\end{array}$ \\
\hline \multirow{2}{*}{ Q 6} & $\mathrm{a}$ & $\begin{array}{l}3 \\
42.9\end{array}$ & $\begin{array}{l}4 \\
57.1\end{array}$ & $\begin{array}{l}0 \\
0.0\end{array}$ & $\begin{array}{l}0 \\
0.0\end{array}$ & $\begin{array}{l}2 \\
28.6\end{array}$ & $\begin{array}{l}3 \\
42.8\end{array}$ & $\begin{array}{l}1 \\
14.3\end{array}$ & $\begin{array}{l}1 \\
14.3\end{array}$ & 17.00 & -1.050 & $\begin{array}{l}0.294 \\
\text { NS }\end{array}$ \\
\hline & $\mathrm{b}$ & $\begin{array}{l}1 \\
14.3\end{array}$ & $\begin{array}{l}2 \\
28.6\end{array}$ & $\begin{array}{l}4 \\
57.1\end{array}$ & $\begin{array}{l}0 \\
0.0\end{array}$ & $\begin{array}{l}1 \\
14.3\end{array}$ & $\begin{array}{l}3 \\
42.8\end{array}$ & $\begin{array}{l}2 \\
28.6\end{array}$ & $\begin{array}{l}1 \\
14.3\end{array}$ & 23.50 & -0.136 & $\begin{array}{l}0.891 \\
\text { NS }\end{array}$ \\
\hline \multirow{2}{*}{ Q 7} & $\mathrm{a}$ & $\begin{array}{l}2 \\
28.6 \\
\end{array}$ & $\begin{array}{l}4 \\
57.1 \\
\end{array}$ & $\begin{array}{l}1 \\
14.3 \\
\end{array}$ & $\begin{array}{l}0 \\
0.0 \\
\end{array}$ & $\begin{array}{l}3 \\
42.9 \\
\end{array}$ & $\begin{array}{l}1 \\
14.2 \\
\end{array}$ & $\begin{array}{l}3 \\
42.9 \\
\end{array}$ & $\begin{array}{l}0 \\
0.0 \\
\end{array}$ & 22.50 & -0.271 & $\begin{array}{l}0.786 \\
\text { NS }\end{array}$ \\
\hline & $\mathrm{b}$ & $\begin{array}{l}2 \\
28.6 \\
\end{array}$ & $\begin{array}{l}3 \\
42.8 \\
\end{array}$ & $\begin{array}{l}2 \\
28.6 \\
\end{array}$ & $\begin{array}{l}0 \\
0.0 \\
\end{array}$ & $\begin{array}{l}3 \\
42.9 \\
\end{array}$ & $\begin{array}{l}3 \\
42.9 \\
\end{array}$ & $\begin{array}{l}1 \\
14.2 \\
\end{array}$ & $\begin{array}{l}0 \\
0.0 \\
\end{array}$ & 19.50 & -0.685 & $\begin{array}{l}0.493 \\
\text { NS }\end{array}$ \\
\hline
\end{tabular}

a: Before denture insertion; b: After denture insertion. NS: Not significant $(p>0.05)$.

The results of follow up of patients to 24 months to asses fracture percentage of the complete dentures showed that, at 18 months, fracture of 4 mandibular complete dentures with vertex soft acrylic liner (Periphery with soft liner $4 \pm 0.5 \mathrm{~mm}$.) and percentage $12.22 \%$ (Table 6 ).

Table (6): Percentage of fracture of complete denture lined with soft liner after 18 months.

\begin{tabular}{lllll}
\hline Sample design & \multicolumn{2}{c}{ Maxillary denture } & \multicolumn{2}{c}{ Mandibular denture } \\
& No. of sample & $\%$ & No. of sample & $\%$ \\
\hline Fracture of denture $2 \pm 0.5$ & 0 & $0 \%$ & 0 & $0 \%$ \\
\hline Fracture of denture $4 \pm 0.5$ & 0 & $0 \%$ & 4 & $12.22 \%$
\end{tabular}




\section{DISCUSSION}

According to the results of this study, the effect of soft denture liners during function is of significance in the prosthodontic treatment of denture patients, and more comfortable. These findings agreed with Kawano et $a l^{(11)}$, ttheoretically, the soft denture liner acts as a damping structure that reduces peak stress as a function of mastication force and also acts as a stress distributor wherein force can be diverted more evenly to other locations in the supporting structures.

Complete dentures are and will remain the mainstay of treatment for the vast majority of edentulous patients; most are satisfied with their dentures but some others are unable to adapt. ${ }^{(12)}$

Resilient denture liner may reduce trauma to mucosa compressed between the underlying bone spicules and a hard denture base. ${ }^{(13)}$

The results according to duration wearing denture with soft liner (6 and 12 months) showed a significant difference for satisfaction with dentures, appearance, and how well other people like their denture. This result agreed with Kiat-Amnuay et al ${ }^{(14)}$ they concluded that, the average service time of a soft liner is 1-2 years. Extension beyond 1 year should be considered.

The results of this study according to flange's length of periphery without hard denture base $2 \pm 0.5 \mathrm{~mm}$. and $4 \pm 0.5 \mathrm{~mm}$. showed significant differences and preferable treatment with $2 \pm 0.5 \mathrm{~mm}$, these findings explained by Kawano et $a l^{(8)}$, lining design that had the soft denture liner extended to the periphery of the denture base demonstrated the greatest shock absorbability of all the tested designs. ${ }^{(8)}$

Higher percentage of denture fracture after 18 months could be due to a plasticized acrylic, was the softest of the group initially but became harder between 115 and 347 days. ${ }^{(14)}$ And other authors concluded that Shore A hardness values of soft liner increased from a low of 9.4 units immediately after fabrication to a maximum of 15.9 units after 1 year. ${ }^{(15)}$

Soft denture liners are used for patients who experience chronic soreness or persistent pain when using conventional dentures as compensation for the reduced ability of the alveolar mucosa to so act. This study demonstrated that soft denture liners reduce the impact force transmitted to the supporting structures and act as damping materials, and that the lining design affects their damping properties. ${ }^{(8)}$

Consequently, the material(s) with the least change in softness and surface roughness with age would be the most clinically applicable. ${ }^{(16)}$

\section{CONCLUSIONS}

Complete denture lined with vertex heat cured material was more comfortable than conventional denture; durability of this material was more than 12 months. Vertex flange periphery without heat cured resin denture base should not more than $2 \mathrm{~mm}$.

\section{REFERENCES}

1. Wright PS. The success and failure of denture soft-lining materials in clinical use. J. Dent. , 1984; 12: 319-327.

2. Qudah, S Harrison A. and Huggett, R. Soft lining materials in prosthetic dentistry: a review. Int. J. Prosthodont.1990; 3: 477783.

3. Mese A, Guzel KG. Effect of storage duration on the hardness and tensile bond strength of silicone- and acrylic resin based resilient denture liners to a processed denture base acrylic resin. $J$ Prosthet Dent. 2008; 99(2):153-159.

4. Jacobsen NL, Mitchell DL, Johnson DL, and Holt RA. Lased and sandblasted denture base surface preparations affecting resilient liner bonding. $J$ Prosthet Dent. 1997; 78:153-158.

5. Botega DM, Sanchez JL, Mesquita MF, Henriques GE, Consani RL. Effects of thermocycling on the tensile bond strength of three permanent soft denture liners. $J$ Prosthodont. 2008; 17:550-554.

6. Keefe JO. Why is my patient experiencing persistent pain after a denture adjustment? JCDA. 2009; 5(75): 359-360.

7. Kawano F., Dootz ER, Koran A., Graig RG. Comparison of bond strength of six soft liners to denture base. $J$ Prosthet Dent. 1992; 2(68): 368-371.

8. Kawano F, Ohguri T, Oran A, Matsumoto $\mathrm{N}$, and Ichikawa $\mathrm{T}$. Influence of lining design of three processed soft denture liners on cushioning effect. $J$ of Oral Rehabil. 1999; 26: 962-968. 
9. Kulkarni RS and Parkhedkar R. The effect of denture base surface pretreatments on bond strengths of two long term resilient liners. J Adv Prosthodont. 2011; 3(1): 1619.

10.Guckes AD, Smith DE, Swoope CC. Counseling and related factors influencing satisfaction with dentures. $J$ Prosth Dent. 1978; 39: 259-67. Cited by Hatim NA, and Jawad IAM: Determination of the occlusal plane in completely edentulous patient by computerized cephalometric method. A Comparative study (Part I). Rafid Dent J. 2006; 6 (Sp. Issue): 1S-9S.

11.Kawano F, Tada N, Nagao K, and Matsumoto $\mathrm{N}$. Influence of soft lining materials on pressure distribution. $J$ Prosthet Dent. 1991; 65: 567.

12.Carlssson GE, and Omar R. The future of complete dentures in oral rehabilitation. $J$ Oral Rehabil. 2010; 37: 143-156
13. O'Keefe J. Why is my patient experiencing persistent pain after a denture adjustment? JCDA. 2009; 5 (75): 359-360).

14.Kiat-Amnuay S, Gettleman L, Mekayarajjananonth T, Khan Z, and Goldsmith J. The influence of water storage on durometer hardness of 5 soft denture liners over time. J Prosthodont. 2005; $14: 19-24$

15.Purr GR, Rueggeberg FA. PhysicalProperty comparison of a chair side- or laboratory-polymerized permanent softliner during 1 year. $J$ Prosthod. 1999; 8:9299.

16.Hermann C, Mesquita MF, and Consani RLX. The effect of aging by thermal cycling and mechanical brushing on resilient denture liner hardness and roughness. J Prosthodont. 2008; 17: 318322 